\title{
Long-Term Functional and Protective Actions of Preconditioning With Hypoxia, Cobalt Chloride, and Desferrioxamine Against Hypoxic-Ischemic Injury in Neonatal Rats
}

\author{
NICOLE M. JONES, LIUBOV KARDASHYAN, JENNIFER K. CALLAWAY, ELIZABETH M. LEE, AND PHILIP M. BEART
}

Brain Injury and Repair Group [N.M.J., L.K., E.M.L., P.M.B], Howard Florey Institute; Department of Pharmacology [N.M.J., J.K.C.], University of Melbourne, VIC 3010 Melbourne, Victoria, Australia

\begin{abstract}
Preconditioning with hypoxia and hypoxia-mimetic compounds cobalt chloride $\left(\mathrm{CoCl}_{2}\right)$ and desferrioxamine (DFX) protects against hypoxic-ischemic $(\mathrm{HI})$ injury in neonatal rat brain. We examined long-term functional and protective actions of preconditioning induced by hypoxia, $\mathrm{CoCl}_{2}$ and $\mathrm{DFX}$ in a neonatal rat model of HI. Postnatal day six rat pups were exposed to preconditioning with hypoxia ( $8 \%$ oxygen) or injections of $\mathrm{CoCl}_{2}$, DFX or saline vehicle and $24 \mathrm{~h}$ later rats underwent $\mathrm{HI}$ or sham surgery. Behavioral tests were performed and at the conclusion of experiments, brains removed for morphologic analyses. HI resulted in a large unilateral lesion in the ipsilateral hemisphere compared with sham control rats. All preconditioning treatments significantly reduced the total lesion volume. Behavioral deficits were observed in HI rats compared with sham controls. The reduction in forelimb grasping strength in HI rats was attenuated by preconditioning with hypoxia, $\mathrm{CoCl}_{2}$ and DFX. HI increased the number of foot faults in a grid-walking test and resulted in forelimb asymmetry in the cylinder test. Only preconditioning with hypoxia reversed all three functional deficits after HI. These findings indicate that preconditioning, especially when induced by hypoxia, has the potential to minimize the morphologic and functional effects of neonatal HI injury. (Pediatr Res 63: 620-624, 2008)
\end{abstract}

$\mathrm{B}$ rain injury in humans, which occurs as a result of an hypoxic-ischemic (HI) episode during the perinatal period, is a major cause of mortality and long-term disability (1). Although there is now a greater understanding of the mechanisms involved in cell death processes associated with HI injury (2), there are still no beneficial therapeutic interventions. Several treatments, which have long-term protective actions in animal models of HI, include brain-derived neurotrophic factor, corticosteroids, glutamate receptor antagonists, and hypothermia $(3,4)$. For a long time, mild stress has been known to "precondition" and protect against a subsequent brain insult (5). Treatments providing protection against subsequent injuries include mild ischemia (6), hyperthermia (7), bacterial lipopolysaccharide $(8)$, and hypoxia $(9,10)$.

Tolerance produced by a mild preconditioning episode of hypoxia has been well investigated, and recent studies have indicated that hypoxia-inducible genes may contribute to tol-

Received October 1, 2007; accepted January 10, 2008.

Correspondence: Nicole M. Jones, Ph.D., Department of Pharmacology, School of Medical Sciences, University of New South Wales, NSW 2052, Sydney, Australia; e-mail: nicole.jones@florey.edu.au

Supported by Neurosciences Victoria - Postdoctoral Fellowship to N.M.J. N.H. and M.R.C. (Australia) - Program Grant (236805) and Fellowship to P.M.B. erance in the rat brain (9-11). Tissue hypoxia can stimulate the expression of many genes involved in adaptive processes such as erythropoiesis, angiogenesis, glucose transport, and anaerobic glycolysis (12). One vital element involved in regulating expression of hypoxia-responsive genes is the transcription factor, hypoxia-inducible factor-1 (HIF-1) (12). In the brain, HIF-1 has been found to have complex roles in brain injury and protective processes, which may relate to insult severity (12). Following exposure to hypoxia or hypoxiamimetic compounds, such as cobalt chloride $\left(\mathrm{CoCl}_{2}\right)$ or desferrioxamine (DFX), HIF- $1 \alpha$ mRNA and protein expression are increased (13). Interestingly, preconditioning with hypoxia, $\mathrm{CoCl}_{2}$ and DFX can prevent against a subsequent HI injury and the protection appears to directly relate to HIF- $1 \alpha$ protein expression (9). These preconditioning treatments do not cause neuronal injury (9), but have been shown to induce changes in gene expression and intracellular signaling pathways $(9-11,14)$. Those genes affected include HIF-1 (9) and HIF-1 target genes: glucose transporters, glycolytic enzymes (10), erythropoietin and vascular endothelial growth factor (VEGF) (11). Numerous experimental studies have highlighted the need to examine long-term functional improvements in addition to histologic outcomes after $\mathrm{HI}$ injury in the neonate $(3,15,16)$. Therefore, we have investigated whether preconditioning with hypoxia (HP), DFX and $\mathrm{CoCl}_{2}$ has long lasting cytoprotective actions and thus improves both morphologic and behavioral endpoints $5 \mathrm{wk}$ after an $\mathrm{HI}$ insult performed in postnatal day 7 rat pups.

\section{METHODS}

All animal work conducted in this study was approved by the Howard Florey Institute Animal Ethics and Experimentation Committee and performed in accordance with the guidelines of the National Health and Medical Research Council (Australia). Sprague-Dawley rat pups were obtained from Animal Resources Centre (Perth, WA) and were housed under standard housing conditions in the Howard Florey Institute animal facility throughout experiments.

Preconditioning treatments. Male and female Sprague-Dawley rat pups (postnatal day 6 (p6)) were randomly exposed to preconditioning treatments (typically two to three different preconditioning treatments were used per litter). Hypoxic preconditioning (HP) was performed as described previously. Briefly, pups $(n=19)$ were exposed to an $8 \% \quad \mathrm{O}_{2} / 92 \% \quad \mathrm{~N}_{2}$ humidified

\footnotetext{
Abbreviations: $\mathbf{C o C l}_{2}$, cobalt chloride; DFX, desferrioxamine; HIF-1, hypoxia-inducible factor-1; HI, hypoxia-ischemia; HP, hypoxic preconditioning; p, postnatal day
} 
atmosphere in $1 \mathrm{~L}$ chambers, which were partially submerged in a water bath maintained at $37^{\circ} \mathrm{C}$ for $3 \mathrm{~h}$. Control rats (no preconditioning) were maintained at $37^{\circ} \mathrm{C}$ for $3 \mathrm{~h}$ in normoxic conditions $(n=8)$. Preconditioning with the hypoxia-mimetic compounds $\mathrm{DFX}$ and $\mathrm{CoCl}_{2}$ was performed as follows. Rat pups received a single s.c. injection on $\mathrm{p} 6$ (up to a maximum volume of 0.1 $\mathrm{mL})$ of one of the following treatments: DFX $(200 \mathrm{mg} / \mathrm{kg} ; n=16)$ or $\mathrm{CoCl}_{2}$ $(60 \mathrm{mg} / \mathrm{kg} ; n=16)$. Vehicle control rats were injected with a single s.c. injection of saline $(0.9 \%$ sterile saline, up to a maximum volume of $0.1 \mathrm{~mL})$ vehicle solution $(n=13)$. Both "nonpreconditioned" control groups (normoxia and vehicle injection) did not differ statistically; therefore, all data for these two groups were combined into the HI alone group $(n=21)$. Following preconditioning treatments, all pups were returned to their dam.

Animal surgery. Twenty-four hours after control or preconditioning treatments, pups were anesthetized with $1.5 \%$ isoflurane in $30 \% \mathrm{O}_{2} / 70 \% \mathrm{~N}_{2}$ mixture and underwent unilateral $\mathrm{HI}$ as described previously (17). The right common carotid artery was exposed through a ventral midline neck incision and permanently occluded by electrocoagulation using a cautery device. The wound was sutured and rats were returned to their mother for 1.5-2 h. Sham control rats $(n=7)$ underwent the identical procedure, without carotid artery occlusion. Pups were then placed in an $8 \% \mathrm{O}_{2} / 92 \% \mathrm{~N}_{2}$ humidified chamber at $37^{\circ} \mathrm{C}$ for $2.5 \mathrm{~h}$. This combined procedure results in select neuronal damage or infarction in the hemisphere ipsilateral to the carotid occlusion, whereas hypoxia alone (contralateral hemisphere) does not produce any significant brain injury (17-19). Following the HI or sham surgery procedure, all pups were returned to their dam and kept under standard housing conditions for the remainder of the study. On p21, pups were weaned and separated out into same-sex cages. Pups were handled every 2-3 d and exposed to behavioral apparatus on the day before testing.

Behavioral testing. Sensorimotor tests were videotaped and behaviors were assessed by an experimenter blinded to the treatment rats had received. All behavioral testing was conducted during the second half of the light

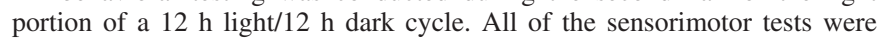
conducted on the same day (p42), whereas locomotor testing was performed on p43 and p44.

Spontaneous locomotor activity. Spontaneous locomotor activity was monitored on $\mathrm{p} 43$ and $\mathrm{p} 44$, using the Truscan system (Coulbourn Instruments, Allentown, USA). Individual rats were placed in locomotor cells and monitored for a single session of $15 \mathrm{~min}$ on each day. Locomotor activity was monitored in horizontal and vertical planes and time spent moving was also measured. Only data from p44 was collected and analyzed.

Grip traction test. A modified grip traction test was used to measure forelimb muscle strength (20). The ability of each animal to hang on to a horizontal rope (plastic tube $-0.6 \mathrm{~cm}$ diameter placed horizontally $50 \mathrm{~cm}$ above table) by the forelimbs was monitored and the time to falling was noted. If a rat had not fallen within $60 \mathrm{~s}$, this was recorded as the maximum time to falling.

Grid-walking test. To measure fore and hind limb deficits, the grid walking test was used (20). Rats were placed on a horizontal grid $(40 \times 40 \mathrm{~cm}$, squares $-2 \times 2 \mathrm{~cm}$, wire $-0.4 \mathrm{~cm}$ diameter). When rats misplaced a limb between the grid squares, a foot fault was counted. The number of foot faults made in 2 min and the total time spent moving were noted. Results are expressed as number of faults/time spent moving (min).

Cylinder test. The cylinder test was used to assess forelimb asymmetry (21). Forelimb bias was analyzed by measuring exploratory (rearing) behaviors of rats placed in a $4 \mathrm{~L}$ transparent cylinder. A mirror was placed behind the cylinder to allow analysis of forelimb movements when rats were facing away from the video camera. The number of forelimb placements on the cylinder wall (ipsilateral, contralateral, "combined"- both paws used simultaneously) made within a 2-min period were counted. Results are expressed as an Asymmetry Score which is calculated as described previously (22). Briefly, the number of ipsilateral touches of the wall plus half the number of "combined" movements is then divided by the total number of forelimb movements (ipsilateral, contralateral, and combined). This gives an asymmetry score-whereby, if there is no asymmetry, the score is 0.5 and an increase in use of the ipsilateral forelimb is indicated by a score $(<0.5)$.

Morphologic staining. After behavioral testing (on p45), all rats were anesthetized with pentobarbitone sodium $(100 \mathrm{mg} / \mathrm{kg}$, i.p), perfused with PBS and brains removed and frozen using isopentane. Brains were subsequently sectioned using a cryostat and $20 \mu \mathrm{m}$ sections collected on gelatin-coated slides stored frozen at $-80^{\circ} \mathrm{C}$ until use. Nissl stained (cresyl violet) sections were examined under a light microscope (Leica DMR). Images were quantified using MCID ${ }^{\mathrm{TM}}$ Analysis (Imaging Research) imaging system to measure left and right hemispheric volume and to calculate lesion volume. Volume was calculated by measuring the area of left and right hemispheres and multiplying by the distance between sections. On average, 18-20 sections per brain were used to calculate lesion volumes. In a similar manner, the volumes of left and right striatum and cortex were measured. All procedures related to lesion volume analysis were performed by an experimenter blinded to the animal treatments. Data are expressed as volume of injured area $\left(\mathrm{mm}^{3}\right.$; volume of left hemisphere - volume of right hemisphere).

Statistics. Statistical analyses were performed using one-way ANOVA, with multiple intergroup comparisons made using Newman-Keuls post hoc test. All data are represented as the mean \pm SEM. A probability value of less than 0.05 was considered statistically significant.

\section{RESULTS}

Similar to our previous findings and those of others using the Rice-Vannucci model of neonatal HI injury, in the present study we observed unilateral brain lesions $(10,17,23)$, which were accompanied by behavioral deficits. There were large lesions affecting the ipsilateral (right) hemisphere in HI rats, including damage to cortical and striatal regions (Fig. 1). Damage in the ipsilateral cortex after HI was reflected by shrinkage of the whole cortex. All cortical layers exhibited damage and in agreement with previous authors, we observed columnar cortical cell loss after HI (Fig. 1B). In the striatum, damage occurred throughout the caudate putamen, predominantly affecting areas close to the lateral ventricle and corpus callosum (Fig. $1 G$ ). Rats preconditioned with $\mathrm{HP}, \mathrm{CoCl}_{2}$ or DFX at $24 \mathrm{~h}$ before HI, exhibited a differential reduction in cell loss throughout the ipsilateral hemisphere in the two specific brain regions examined (Fig. 1). The total volume of brain damage at $5 \mathrm{wk}$ after surgery was significantly increased in $\mathrm{HI}$ animals $\left(67.8 \pm 14.0 \mathrm{~mm}^{3}\right)$ compared with control rats $\left(1.4 \pm 4.7 \mathrm{~mm}^{3}\right)(\dagger p<0.05)$. A marked reduction in total lesion volume occurred in all groups of rats exposed to preconditioning treatments $24 \mathrm{~h}$ before $\mathrm{HI}$ surgery. $\mathrm{HP}, \mathrm{CoCl}_{2}$ and DFX reduced overall lesion volume by similar amounts: $68 \%, 68 \%$, and $53 \%$, respectively, compared with rats exposed to $\mathrm{HI}$ alone (Fig. 2). Preconditioning with hypoxia and $\mathrm{CoCl}_{2}$ produced appreciable reductions in the extent of cortical damage and only HP significantly reduced striatal damage. There was a trend for $\mathrm{CoCl}_{2}$ to reduce damage in the striatum, but this was not significant and DFX preconditioning had no effect on striatal damage compared with $\mathrm{HI}$ alone.

At 5 wk after HI, rats exhibited several sensorimotor behavioral deficits when compared with control rats, which have been previously described $(4,20)$. Rats subjected to HI displayed significantly reduced forelimb muscle strength as assessed using the grip traction test compared with sham control rats. These animals were able to hold on to the rope for $17 \pm$ $3 \mathrm{~s}$, whereas control rats lasted $36 \pm 5 \mathrm{~s}$ before falling. There was a significant improvement in the forelimb muscle strength in all of the preconditioning groups when compared with $\mathrm{HI}$ alone. Rats exposed to preconditioning with $\mathrm{HP}, \mathrm{CoCl}_{2}$, and DFX were able to hold on to the rope for $1.8,1.8$, and 2.4 times longer than $\mathrm{HI}$ alone group, respectively (Fig. $3 A$ ).

The number of foot faults made was assessed using the grid walking test and $\mathrm{HI}$ rats $(13.6 \pm 1.4$ mistakes $/ \mathrm{min})$ made more mistakes compared with control rats $(9.5 \pm 1.1$ mistakes $/ \mathrm{min})$. Only HP significantly reduced the number of foot faults made after $\mathrm{HI}$ to $8.3 \pm 0.9$ mistakes $/ \mathrm{min}$. $\mathrm{CoCl}_{2}$ and $\mathrm{DFX}$ preconditioning did not reduce the number of foot faults made (Fig. 3B).

The cylinder test is used to assess forelimb asymmetry in models of unilateral brain injury $(21,22)$. Control rats had an 

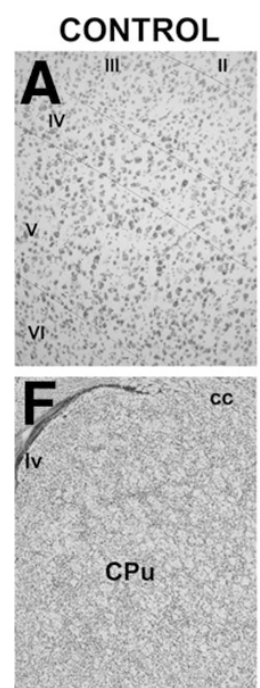

$\mathrm{HI}$
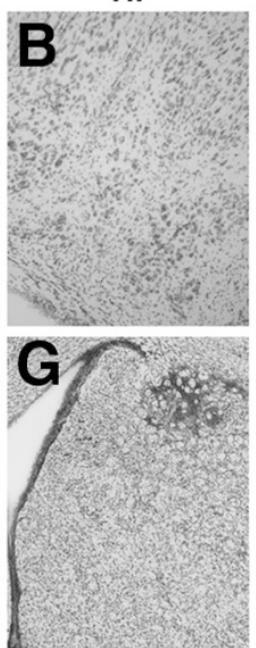

$\mathrm{HP}+\mathrm{HI}$
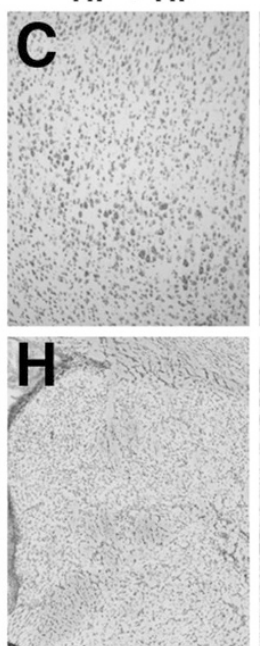

$\mathrm{CoCl}_{2}+\mathrm{HI}$
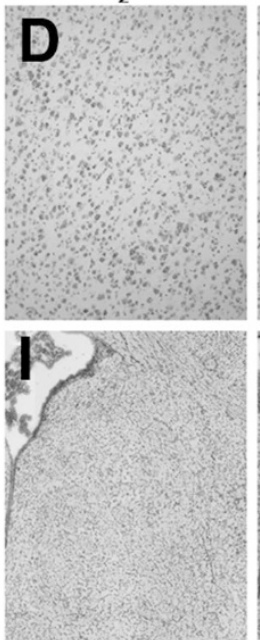

\section{$\mathrm{DFX}+\mathrm{HI}$}
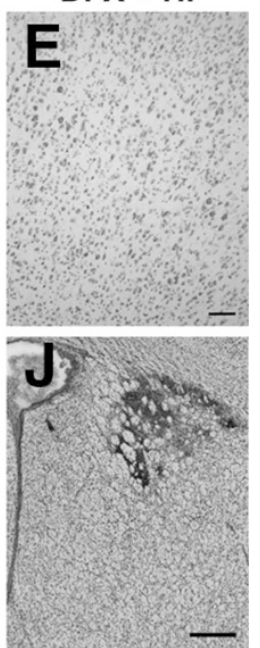

Figure 1. Photomicrographs demonstrating the protective effects of preconditioning with hypoxia (HP), $\mathrm{CoCl}_{2}$, and DFX at 5 wk after $\mathrm{HI}$. On postnatal day 6 (p6), rats were preconditioned with $\mathrm{HP}, \mathrm{CoCl}_{2}$, or DFX and on p7, subjected to an $\mathrm{HI}$ insult. Brains were collected at 5 wk after $\mathrm{HI}$ and coronal brain sections were stained with cresyl violet to illustrate damage to cortex $(A-E)$ and striatum $(F-J)$. HI resulted in damage to the cortex $(B)$ throughout cortical layers (II-VI) compared with control $(A)$. HP, $\mathrm{CoCl}_{2}$, and DFX preconditioning treatments reduced the extent of cortical damage after $\mathrm{HI}(C-E)$. In the striatum, $\mathrm{HI}(G)$ produced damage throughout caudate putamen $(\mathrm{CPu})$, predominantly affecting areas adjacent to the lateral ventricle (lv) and corpus callosum (cc) compared with control $(F)$. HP and $\mathrm{CoCl}_{2}$ preconditioning $(H, I)$ appeared to improve cell survival in striatum, whereas DFX had no effect $(J)$. Scale bar $=100 \mu \mathrm{m}(A-E)$; 500 $\mu \mathrm{m}(F-J)$.
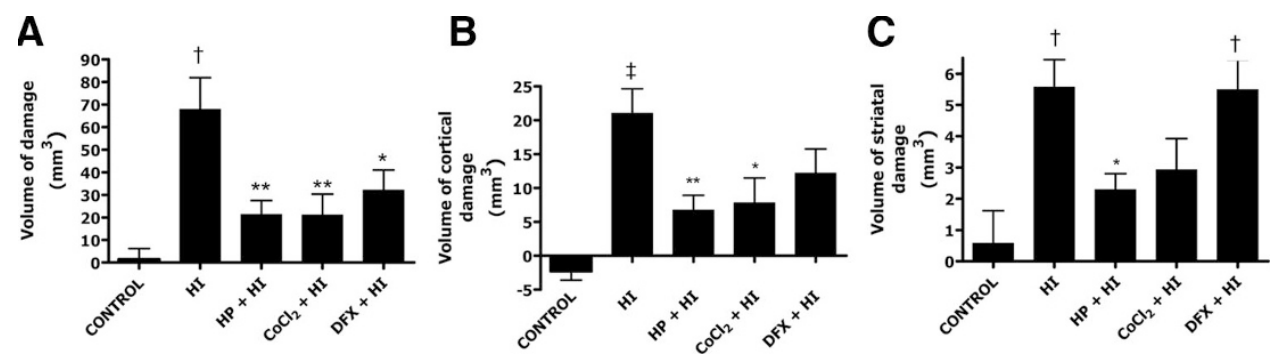

Figure 2. Quantification of brain damage at $5 \mathrm{wk}$ after HI injury. (A) HI $(n=21)$ significantly increased the total volume of brain damage compared with sham control rats $(n=7)$. Preconditioning with hypoxia $(n=19), \mathrm{CoCl}_{2}(n=16)$, and DFX $(n=16)$ significantly reduced the total amount of brain damage after $\mathrm{HI}(\dagger p<0.05$, compared with control rats; $* p<0.05$, **p $<0.01$, compared with vehicle treated HI rats). HI significantly increased the volume of $(B)$ cortical and $(C)$ striatal brain damage compared with control rats $(\dagger p<0.05, \ddagger p<0.01)$. (B) $\mathrm{HP}$ and $\mathrm{CoCl}_{2}$ reduced the amount of cortical injury $(* p<0.05)$, while only $(C) \mathrm{HP}$ reduced the amount of striatal injury compared with vehicle treated HI rats. Data are shown as the mean \pm SEM.
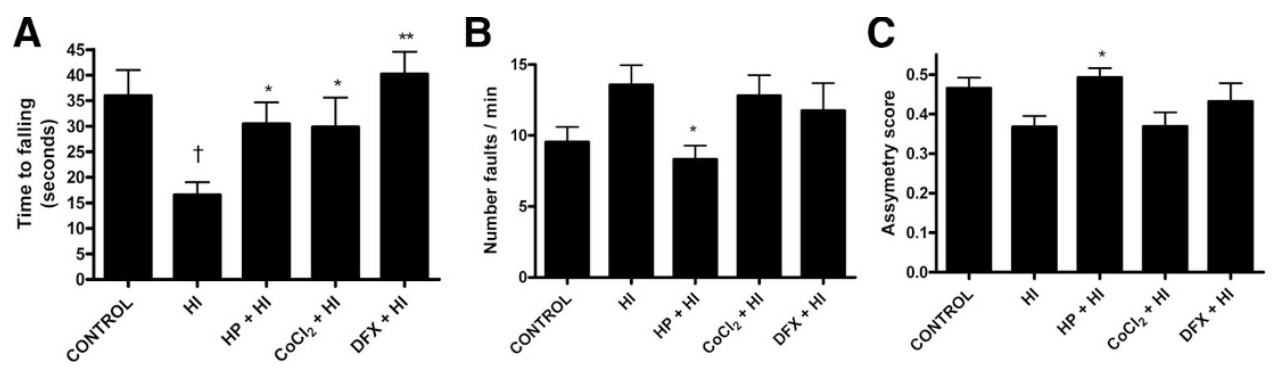

Figure 3. Preconditioning improves behavioral deficits up to $5 \mathrm{wk}$ after HI. $(A) \mathrm{HI}(n=21)$ reduces forelimb muscle strength compared with control ( $\dagger p<$ $0.05)$, as measured by the grip traction test. Preconditioning with hypoxia $(n=19), \mathrm{CoCl}_{2}(n=16)$ and DFX $(n=16)$ significantly improved forelimb muscle strength $(* p<0.05, * * p<0.01)$ compared with HI treatment alone. $(B)$ There was an increase in the number of foot faults made in the grid-walking task in $\mathrm{HI}$ rats compared with control. HP significantly reduced the number of foot faults made $\left({ }^{*} p<0.05\right)$, compared with HI treatment alone. (C) There is a forelimb asymmetry observed in HI rats compared with controls, and this is reversed by HP $\left(^{*} p<0.05\right)$.

asymmetry score of $0.46 \pm 0.03$ indicating that there was no limb preference $(\sim 50 \%$ use of left and right forelimbs) for exploring the cylinder. HI rats had an asymmetry score of $0.36 \pm 0.03$, indicating that they prefer using the nonaffected (ipsilateral) forelimb when exploring the cylinder. Preconditioning with hypoxia prevented the ipsilateral forelimb pref- erence observed after $\mathrm{HI}$, with rats having an asymmetry score of $0.49 \pm 0.02$. $\mathrm{CoCl}_{2}$ and DFX failed to exert significant effects on forelimb asymmetry after HI (Fig. 3C).

Locomotor activity has previously been shown to be altered at various times after neonatal HI injury $(24,25)$. We did not observe any significant changes in locomotor activity (hori- 
zontal and vertical movements or time spent moving) with $\mathrm{HI}$ treatment compared with control or between any of the preconditioning treatments at $5 \mathrm{wk}$ after HI (data not shown).

\section{DISCUSSION}

The present study was undertaken to determine the effectiveness of various preconditioning treatments on functional outcomes after HI injury in rats in addition to morphologic outcomes. We have shown that preconditioning with HP, DFX, and $\mathrm{CoCl}_{2}$ can improve some long-term functional deficits and also reduce the total lesion size after HI. HP and $\mathrm{CoCl}_{2}$ preconditioning were able to reduce the extent of cortical injury and only HP reduced striatal damage. Although many treatments have been tested in the HI model at short term intervals, only a few have been examined and found to yield long-term functional outcomes $(3,16,26,27)$. Using preconditioning with hypoxia and two hypoxia-mimetic compounds, $\mathrm{CoCl}_{2}$ and $\mathrm{DFX}$, we observed long-term protective actions of these treatment strategies. One possible mechanism involved in the neuroprotection described in the present study is likely to be the induction of the transcription factor HIF-1, its many target genes and adaptive processes (12).

Previous studies have found that HI causes a number of functional deficits including slowing of various developmental milestones (eye opening, righting reflex), locomotor activity and sensorimotor and memory deficits $(24,25,28,29)$. The present study used three different functional indices to assess behavioral deficits after $\mathrm{HI}$ and of these, the grip traction test which is used to assess forelimb muscle strength, appeared to be the most useful index relating to total lesion volume. The grip traction test has previously been used to test forelimb placing and muscle strength after injury $(20,30)$. Bona et al. (20) used the grip traction test $5 \mathrm{wk}$ after rats underwent HI on p7 and observed a deficit in forelimb muscle strength, which was accompanied by a large ipsilateral lesion. We observed a similar deficit in the grip traction test after HI, which was reversed by all of the preconditioning treatments used and this functional improvement was accompanied by a reduction in total lesion volume.

The cylinder test has been widely used in various adult and neonatal models of unilateral brain injury to assess forelimb asymmetry $(21,22)$, but in our hands, a mild deficit was observed at 5 wk after HI compared with sham control rats. Additionally, we found a small increase in the number of foot faults made in the grid-walking test. Compared with previous studies employing the HI model to examine behavioral deficits, we seem to have used a slightly milder injury ( $\sim 35 \%$ of the ipsilateral hemisphere is damaged, compared with 50$60 \%$ in other studies (15)), which may explain why not all of the behavioral deficits were different in HI animals compared with controls. Indeed, in adult rodents, small lesions produce mild deficits in sensorimotor tasks (forelimb asymmetry, reaching task, grid walking task) compared with a larger lesions (31). Previously, a correlation between the extent of damage in cortex or striatum and forelimb asymmetry has been shown in the rat HI model (32). Indeed, lesions to the ventrolateral regions of the caudate putamen can impair initi- ation of stepping movements, sensorimotor orientation, and skilled motor behavior in rats and to impair motor coordination $(33,34)$. Such movements are required to perform the grid walking and cylinder tests. Nonetheless, we were able to show that preconditioning with hypoxia could improve function in cylinder and grid walking tasks and reduce cortical and striatal damage after HI.

We did not detect any changes in locomotor activity at $5 \mathrm{wk}$ after HI, which is in agreement with the findings of Bona et al. (20). Previous studies have reported that HI can cause changes in spontaneous locomotor activity, at different ages after HI (24). HI only appears to result in an increase in locomotor activity in young rats (postweaning $\sim \mathrm{p} 21$ ), and returns to normal at a later age (p90). However, when the injury is severe enough, hyperactivity can persist into adulthood (25).

HP has previously been shown to improve functional recovery after $\mathrm{HI}$ in the cylinder test and memory test (15), with protection being observed in cortex, striatum, and hippocampus. In the present study, we confirmed that HP could reverse limb asymmetry in the cylinder test and also found that HP can preserve forelimb muscle strength and reduce the number of foot faults made after HI. Thus, the neuroprotective and functional improvements resulting from HP are long lasting, and not simply because of a delay in cell death processes.

The protective effects of preconditioning with $\mathrm{HP}, \mathrm{CoCl}_{2}$, and DFX have previously been shown to relate to their ability to increase HIF-1 (9), but could also involve other mechanisms such as their ability to penetrate the blood brain barrier (BBB) or effects on brain vasculature and cerebral blood flow. Although hypoxia reversed all of the functional deficits after $\mathrm{HI}$, preconditioning with $\mathrm{CoCl}_{2}$ and DFX only improved function in the grip traction test. Both hypoxia-mimetics reduced total lesion volume, but only $\mathrm{CoCl}_{2}$ was able to reduce the size of the cortical lesion produced by HI. Cobalt ions have been found to readily cross the BBB in rodents and humans $(35,36)$. In addition, studies have shown that $\mathrm{CoCl}_{2}$ can induce protective effects in the brain and possible neuroprotective mechanisms involve the production of HIF-1 and HIF-1 target genes (erythropoietin, VEGF) and other protective proteins including heme oxygenase- 1 and metallothionein $(10,13,37)$. Although we did observe a robust histologic protective effect of $\mathrm{CoCl}_{2}$ preconditioning against total lesion volume and cortical injury in the present study, the grip traction test was the only functional test that was improved by $\mathrm{CoCl}_{2}$ treatment. Recently, $\mathrm{CoCl}_{2}$ was shown to produce in vivo brain protection against hypobaric hypoxia (37); however, the present study is the first to indicate that $\mathrm{CoCl}_{2}$ can produce a long-term functional and histologic protection up to $5 \mathrm{wk}$ after $\mathrm{HI}$ injury.

Preconditioning with DFX improved forelimb muscle strength and reduced total lesion volume, but did not reduce the other two behavioral deficits measured after HI. When DFX is administered before insults, it appears to produce a mild protective effect against neonatal HI (9). Interestingly, when DFX is given after an injury, when the BBB is open, greater neuroprotection is observed against cerebral ischemia in neonatal (38) and adult rats (39). Brain levels of DFX have been measured in neonatal rats and represent only $\sim 65 \%$ of the levels present in the brains of HI-injured animals (40). 
More recently, DFX was shown in rats to induce long term functional improvements after middle cerebral artery occlusion (41) and these protective actions are thought to be mediated by HIF-1 and VEGF. In agreement with our own data demonstrating that DFX does not protect the striatum, long-term postinjury administration of DFX was unable to reduce striatal injury after middle cerebral artery occlusion (41). We have demonstrated the long-term neuroprotective properties of treatments, which activate the HIF-1 pathway and further evaluation of the mechanism of action of these agents and related compounds are likely to be incisive.

\section{REFERENCES}

1. van Handel M, Swaab H, de Vries LS, Jongmans MJ 2007 Long term cognitive and behavioural consequences of neonatal encephalopathy following perinatal asphyxia: a review. Eur J Pediatr 166:645-654

2. Johnston MV, Trescher WH, Ishida A, Nakajima W 2001 Neurobiology of hypoxicischemic injury in the developing brain. Pediatr Res 49:735-741

3. Amli C, Levy TJ, Han BH, Shah AR, Gidday JM, Holtzman DM 2000 BDNF protects against spatial memory deficits following neonatal hypoxia-ischemia. Exp Neurol 166:99-114

4. Bona E, Hagberg H, Loberg E, Bagenholm R, Thoresen M 1998 Protective effects of moderate hypothermia after neonatal hypoxia-ischemia: short- and long-term outcome. Pediatr Res 43:738-745

5. Gidday JM 2006 Cerebral preconditioning and ischaemic tolerance. Nat Rev Neurosci 7:437-448

6. Simon RP, Niiro M, Gwinn R 1993 Prior ischemic stress protects against experimental stroke. Neurosci Lett 163:135-137

7. Ota A, Ikeda T, Xia X, Xia Y, Ikenoue T 2000 Hypoxic-ischemic tolerance induced by hyperthermic pretreatment in newborn rats. J Soc Gynecol Investig 7:102-105

8. Eklind S, Mallard C, Arvidsson P, Hagberg H 2005 Lipopolysaccharide induces both a primary and a secondary phase of sensitization in the developing rat brain. Pediatr Res 58:112-116

9. Bergeron M, Gidday JM, Yu AY, Semenza GL, Ferriero DM, Sharp FR 2000 Role of hypoxia-inducible factor-1 in hypoxia-induced ischemic tolerance in neonatal rat brain. Ann Neurol 48:285-296

10. Jones NM, Bergeron M 2001 Hypoxic preconditioning induces changes in HIF-1 target genes in neonatal rat brain. J Cereb Blood Flow Metab 21:1105-1114

11. Bernaudin M, Tang Y, Reilly M, Petit E, Sharp FR 2002 Brain genomic responses following hypoxia and re-oxygenation in neonatal rat. J Biol Chem 277:3972839738

12. Sharp FR, Bernaudin M 2004 HIF1 and oxygen sensing in the brain. Nat Rev Neurosci 5:437-448

13. Zaman K, Ryu H, Hall D, O’Donovan K, Lin KI, Miller MP, Marquis JC, Baraban JM, Semenza GL, Ratan RR 1999 Protection from oxidative stress-induced apoptosis in cortical neuronal cultures by iron chelators is associated with enhanced DNA binding of hypoxia-inducible factor-1 and ATF-1/CREB and increased expression of glycolytic enzymes, p21waf1/cip1, and erythropoietin. J Neurosci 19:9821-9830

14. Jones NM, Bergeron M 2004 Hypoxia-induced tolerance in neonatal rat brain involves enhanced ERK1/2 signalling. J Neurochem 89:157-167

15. Gustavsson M, Anderson M, Mallard C, Hagberg H 2005 Hypoxic preconditioning confers long-term reduction of brain injury and improvement of neurological ability in immature rats. Pediatr Res 57:305-309

16. Balduini W, De Angelis V, Mazzoni E, Cimino M 2001 Simvastatin protects against long-lasting behavioural and morphological consequences of neonatal hypoxic/ ischemic brain injury. Stroke 32:2185-2191

17. Rice JE III, Vannucci RC, Brierley JB 1981 The influence of immaturity on hypoxic-ischemic brain damage in the rat. Ann Neurol 9:131-141

18. Vannucci RC, Towfighi J, Vannucci SJ 1998 Hypoxic preconditioning and hypoxicischemic brain damage in the immature rat: pathologic and metabolic correlates. J Neurochem 71:1215-1220
19. Nakajima W, Ishida A, Lange M, Gabrielson K, Wilson M, Martin L, Blue M, Johnston M 2000 Apoptosis has a prolonged role in the neurodegeneration after hypoxic ischemia in the newborn rat. J Neurosci 20:7994-8004

20. Bona E, Johansson B, Hagberg H 1997 Sensorimotor function and neuropathology five to six weeks after hypoxia-ischemia in seven-day-old rats. Pediatr Res 42:678683

21. Schallert T, Fleming S, Leasure J, Tillerson J, Bland S 2000 CNS plasticity and assessment of forelimb sensorimotor outcome in unilateral rat models of stroke, cortical ablation, parkinsonism and spinal cord injury. Neuropharmacology 39:777787

22. Woodlee MT, Asseo-Garcia AM, Zhao X, Liu SJ, Jones TA, Schallert T 2005 Testing forelimb placing "across the midline" reveals distinct, lesion-dependent patterns of recovery in rats. Exp Neurol 191:310-317

23. Vannucci RC, Christensen MA, Yager JY 1993 Nature, time-course, and extent of cerebral edema in perinatal hypoxic-ischemic brain damage. Pediatr Neurol 9:29-34

24. Balduini W, De Angelis V, Mazzoni E, Cimino M 2000 Long-lasting behavioural alterations following a hypoxic/ischemic brain injury in neonatal rats. Brain Res $859: 318-325$

25. McAuliffe JJ, Miles L, Vorhees CV 2006 Adult neurological function following neonatal hypoxia-ischemia in a mouse model of the term neonate: water maze performance is dependent upon separable cognitive and motor components. Brain Res 1118:208-221

26. Chang YC, Tzeng SF, Yu L, Huang AM, Lee HT, Huang CC, Ho CJ 2006 Early-life fluoxetine exposure reduced functional deficits after hypoxic-ischemia brain injury in rat pups. Neurobiol Dis 24:101-113

27. Noh MR, Kim SK, Sun W, Park SK, Choi HC, Lim JH, Kim IH, Kim HJ, Kim H, Eun BL 2006 Neuroprotective effect of topiramate on hypoxic ischemic brain injury in neonatal rats. Exp Neurol 201:470-478

28. Lubics A, Reglodi D, Tamas A, Kiss P, Szalai M, Szalontay L, Lengvari I 2005 Neurological reflexes and early motor behavior in rats subjected to neonatal hypoxicischemic injury. Behav Brain Res 157:157-165

29. Young RS, Kolonich J, Woods CL, Yagel SK 1986 Behavioral performance of rats following neonatal hypoxia-ischemia. Stroke 17:1313-1316

30. DeGraba TJ, Ostrow P, Hanson S, Grotta JC 1994 Motor performance, histologic damage, and calcium influx in rats treated with NBQX after focal ischemia. J Cereb Blood Flow Metab 14:262-268

31. Hsu JE, Jones TA 2006 Contralesional neural plasticity and functional changes in the less-affected forelimb after large and small cortical infarcts in rats. Exp Neurol 201:479-494

32. Grow JL, Liu YQ, Barks JD 2003 Can lateralizing sensorimotor deficits be identified after neonatal cerebral hypoxia-ischemia in rats? Dev Neurosci 25:394-402

33. Cousins MS, Salamone JD 1996 Involvement of ventrolateral striatal dopamine in movement initiation and execution: a microdialysis and behavioural investigation. Neuroscience 70:849-859

34. Carli M, Evenden JL, Robbins TW 1985 Depletion of unilateral striatal dopamine impairs initiation of contralateral actions and not sensory attention. Nature 313:679682

35. Stevens H, Jansen HM, De Reuck J, Lemmerling M, Strijckmans K, Goethals P, Lemahieu I, De Jong BM, Willemsen AT, Korf J 1999 55Cobalt (Co) as a PET-tracer in stroke, compared with blood flow, oxygen metabolism, blood volume and gadolinium-MRI. J Neurol Sci 171:11-18

36. Badr GA, Zhang J-Z, Tang J, Kern TS, Ismail-Beigi F 1999 Glut1 and Glut3 expression, but not capillary density, is increased by cobalt chloride in rat cerebrum and retina. Brain Res Mol Brain Res 64:24-33

37. Shrivastava K, Shukla D, Bansal A, Sairam M, Banerjee PK, Ilavazhagan G 2008 Neuroprotective effect of cobalt chloride on hypobaric hypoxia-induced oxidative stress. Neurochem Int 52;368-375

38. Mu D, Chang YS, Vexler ZS, Ferriero DM 2005 Hypoxia-inducible factor 1alpha and erythropoietin upregulation with deferoxamine salvage after neonatal stroke. Exp Neurol 195:407-415

39. Liachenko S, Tang P, Xu Y 2003 Deferoxamine improves early postresuscitation reperfusion after prolonged cardiac arrest in rats. J Cereb Blood Flow Metab 23:574-581

40. Palmer C, Roberts RL, Bero C 1994 Deferoxamine posttreatment reduces ischemic brain injury in neonatal rats. Stroke 25:1039-1045

41. Freret T, Valable S, Chazalviet L, Saulnier R, Mackenzie E, Petit E, Bernaudin M, Boulouard M, Schumann-Bard P 2006 Delayed administration of deferoxamine reduces brain damage and promotes functional recovery after transient focal cerebral ischemia in the rat. Eur J Neurosci 23:1757-1765 